\title{
CT guided lung biopsy: Diagnostic yield and complications, using 18G coaxial semi-automatic core needle
}

\author{
Ajit Thapa ${ }^{1}$, Kamal Subedi ${ }^{2}$, Sundar Suwal ${ }^{3}$, Dinesh Chataut ${ }^{4}$ \\ ${ }^{1}$ Teaching Assistant, Department of Radiology and Imaging, Tribhuvan University Teaching \\ Hospital, Kathmandu, Nepal. \\ 2,3,4 Assistant Professor, Department of Radiology and Imaging, Tribhuvan University \\ Teaching Hospital, Kathmandu, Nepal.
}

Received: October 21, 2018

Accepted: November 28, 2018

Published: January 30, 2019

Cite this paper:

Thapa A, Subedi K, Suwal S, Chataut D, et al. CT guided lung biopsy: Diagnostic yield and complications, using 18G coaxial semi-automatic core needle. Nepal Journal of Medical Sciences 2019;4(1):20-25.

\begin{abstract}
Introduction: CT guided biopsy is essential for histopathological diagnosis of suspicious lung nodule, which are not amenable for either bronchoscopic or sonography guided sampling.

Methods: Twenty eight patients with suspicious lung nodules not amenable for bronchoscopic or sonography guided sampling who underwent CT guided lung biopsy with $18 \mathrm{G}$ coaxial semiautomatic core biopsy needle in one year were retrospectively studied for diagnostic yield and complications.

Results: Out of 28 patients, who underwent CT guided lung nodule biopsy, 18 were male and 10 were female. The age ranged from 22 to 80 years. Lesion size ranged from one $\mathrm{cm}$ to four $\mathrm{cm}$ and depth of lesion from pleura ranged from zero $\mathrm{cm}$ to five $\mathrm{cm}$. Diagnostic yield of our core needle biopsy was 26(92.3\%). Clinically significant complication was low. Massive pneumothorax which needed intercostal drainage was $7.14 \%$ (2 patients). Pulmonary hemorrhage manifesting as hemoptysis was seen in $14.3 \%$ ( 2 patients). No hemothorax or air embolism was noted in any of the patient.
\end{abstract}

Conclusion: CT guided lung lesion biopsy with $18 \mathrm{G}$ coaxial semi-automatic core biopsy needle is a safe procedure with good diagnostic yield and relatively low incidence of clinically significant complications.

Key words: CT guided lung biopsy; complications; diagnostic yield; pneumothorax

Correspondence: Ajit Thapa, Teaching Assistant, Tribhuvan University Teaching Hospital, Kathmandu, Nepal.

Email address: drajitthapa@gmail.com

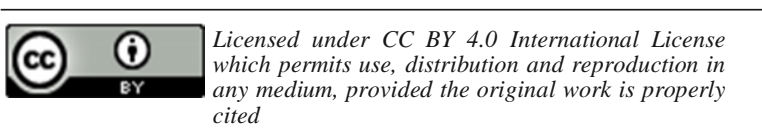




\section{INTRODUCTION}

Lung cancer is the most common cancer in the world and is the leading cause of cancer related death worldwide.[1] Central lung cancer with airway obstruction is generally sampled bronchoscopically, whereas peripheral lung lesions abutting chest wall is sampled under sonographic guidance. $[2,3]$ Computer tomography (CT) guided lung biopsy is reserved for lesions with intervening normal lung between mass and chest wall or lesions difficult to visualize on sonography due to intervening bone or paravertebral location

Compared to diagnostic yield of fine needle aspiration cytology (FNAC) of 88\%; biopsy has higher diagnostic yield up to 94\%.[4].

\section{METHODS}

Ethical clearance for the retrospective study was taken from ethical board of the hospital. This study was conducted in Department of Radiology and Imaging, Tribhuvan University Teaching hospital. All patients with suspected lesion on Contrast enhanced CT, not amenable for bronchoscopic or sonographic sampling and underwent CT guided lung biopsy from September 2017 to October 2018 were searched from medical record and included in this study. Images were retrieved from CT console. Medical history, complication and histopathological reports were obtained from the medical record. Parameters like size of nodule, depth i.e. distance from pleural puncture to lesion margin were measured from $\mathrm{CT}$ images retrieved from console.

\section{Biopsy Procedure}

Patients with platelet count of less than 50,000 and INR of more than 1.5 were excluded from the study. All CT guided biopsy were performed in 64 slice CT scanner with reconstructed slice thickness of two mm. The $18 \mathrm{G}, 15 \mathrm{~cm}$ long semi automatic core biopsy needle with $18 \mathrm{~mm}$ sampling notch with its coaxial needle were used in all biopsies. Patient were kept nil per oral for four hours prior to procedure. On planning CT, the shortest, safest route without fissure was chosen. Procedure was done under local anaesthesia, both skin and pleura were anesthetized using $2 \%$ lignocaine. Attempt were made to puncture pleura as perpendicular as possible and not to emphysematous bulla, fissure and large (pulmonary vessels with diameter more than two mm). After targeting the lesion with $17 \mathrm{G}$ coaxial needle, multiple (at least three) cores were taken with coaxially introduced semi automatic biopsy needle and secured in 10 percent formalin solution. Intravenous tranexamic acid at dose of $500 \mathrm{mg}$ to $1000 \mathrm{mg}$ was given in case of significant haemoptysis. Check scan was done after five minutes of biopsy and repeated if pneumothorax was detected. Significant increase in pneumothorax was drained under water seal with $8 \mathrm{~F}$ pigtail catheter. Minimal to mild pneumothorax were conservatively managed with high flow oxygen and check radiography at four hours.

All the data and images were studied to calculate the diagnostic yield and incidence of complications.

\section{RESULTS}

From September 2017 to August 2018, there were total of $28 \mathrm{CT}$ guided suspicious lung nodule biopsies in our institute. There were 18 male and the age ranged from 22 to 80 years. The sizes of nodule sampled varied from one to four $\mathrm{cm}$ with mean size of $2.6 \mathrm{~cm}$. Depth of nodule from pleura ranged from zero to five $\mathrm{cm}$. Ten nodules were in left lower lobe, seven in left upper lobe, five in right upper lobe and three each 
in right middle and lower lobe, as in Table 1.

Fourteen biopsies showed adenocarcinoma on histopathology. Similarly, squamous cell carcinoma and small cell carcinoma were seen in six and four biopsies, respectively. Two pulmonary nodules showed granuloma. However, in two biopsies fibrosis was seen on histology, which was not consistent with the CT findings, as in Table 2. Out of 28, 26 patients showed definite diagnosis on histology, averaging our diagnostic yield to $92.3 \%$.

In our observational study, total significant complication was $25 \%$, summarized in Table 3. Pulmonary haemorrhage evidenced by perilesional ground glass opacity were seen in $11(39 \%)$ patients of which $4(14 \%)$ had haemoptysis, as in figure 1D. Two were minimal which was self-limiting and two were conservatively managed with intravenous anti fibrinolytic like tranexamic acid. All four patients with haemoptysis were having deep lesions with depth of more than three $\mathrm{cm}$ from the pleura.

Pneumothorax occurred in eight patients $(28 \%)$ out of which five $(17 \%)$ were minimal pneumothorax which did not progressed on repeat scan at five minutes, as depicted in figure 1C. One patient had mild pneumothorax which was aspirated using the same coaxial needle in the same sitting. Check chest radiography after four hours showed no pneumothorax. However, two patients with deep lesions (7.1\%) developed massive pneumothorax which needed drainage and needed overnight observation, as in Figure 2.

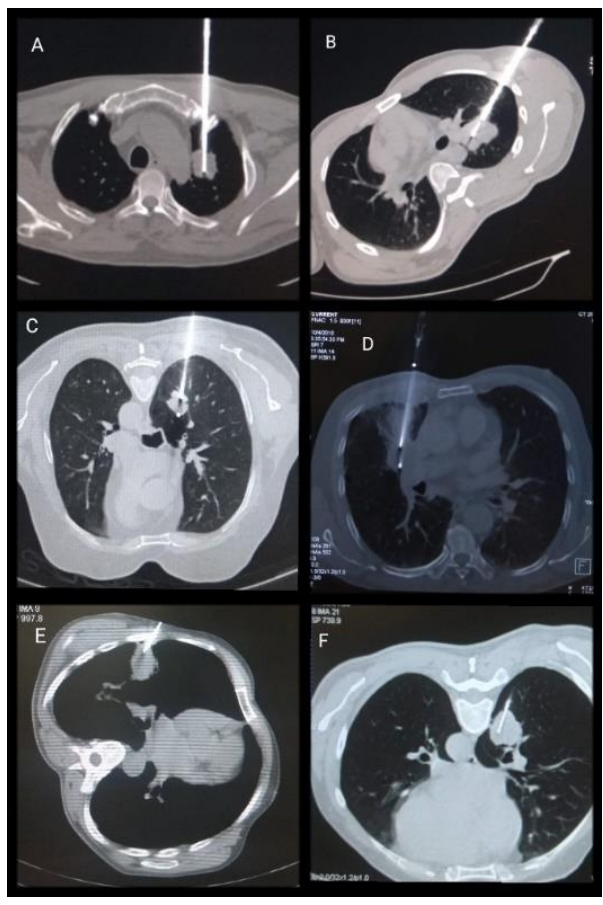

Figure 1.

A. Left upper lobe lung nodule abutting aortic arch, Biopsy under CT guidance. Histology showed small cell carcinoma.

B. Central lung mass in left upper lobe, CT guided biopsy proved adenocarcinoma. Non progressive minimal pneumothorax developed during biopsy.

C. CT guided biopsy of $2.2 \mathrm{~cm}$ lung nodule with minimal pneumothorax during the procedure, No further increase in pneumothorax. Histopathology showed adenocarcnoma.

D. CT guided biopsy of central lung mass which developed perilesional hemorrhage. HPE showed fibrotic tissue.

E. CT guided biopsy of peripheral lung nodule deep to rib. HPE showed adenocarcinoma.Right lower lobe lung nodule biopsied under CT guidance. Minimal post biopsy pneumothorax. Histology was consistent with squamous cell carcinoma.

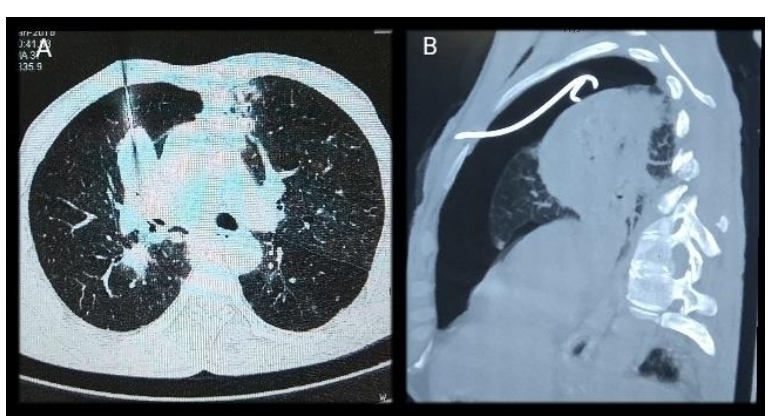

Figure 2.

A. CT guided biopsy of central right middle lobe lung mass with

B. Massive pneumothorax after biopsy which was drained with pigtail drainage catheter. 
Table 1. Characteristic of patients and lesions

\begin{tabular}{lll}
\hline Characteristics of patient and lesion & Data \\
\hline Sex & Male & 18 \\
& Female & 10 \\
Age & Mean \pm SD & $59.07 \pm 13.08$ \\
& Range & years \\
Lesion Size & Mean & $22-80$ years \\
Depth from pleura & Range & $2.6 \mathrm{~cm}$ \\
\multirow{3}{*}{ Lesion Location } & Mean & $1-4 \mathrm{~cm}$ \\
& Range & $1.5 \mathrm{~cm}$ \\
& Right upper lobe & $0-5$ \\
& Right middle lobe & 5 \\
& Right lower lobe & 3 \\
& Left upper lobe & 3 \\
& Left lower lobe & 7 \\
\hline
\end{tabular}

Table 2. Histopathology results

\begin{tabular}{lll}
\hline Histopathology & Adenocarcinoma & 14 \\
& Squamous cell carcinoma & 6 \\
Small cell carcinoma & 4 \\
& Inflammatory & 2 \\
& Inconsistent with CT finding & 2 \\
\hline
\end{tabular}

Data presented as frequency

Table 3. Complications

\begin{tabular}{lll}
\hline Complication & & Total \\
\hline Pneumothorax & Minimal & 5 \\
& Mild & 1 \\
& Need drainage & 2 \\
Pulmonary hemorrhage & No Hemoptysis & 7 \\
New Perilesional opacity & Hemoptysis Total & 4 \\
& Hemoptysis(Controlled with medication & 2 \\
Hemothorax & & 0 \\
Air embolism & & 0 \\
\hline
\end{tabular}

Data presented as frequency 


\section{DISCUSSION}

Lung cancer is the most common cancer worldwide and is leading cause of cancer related death.[1] Management of suspicious pulmonary lesions needs accurate histopathology diagnosis which can be done with bronchoscopy, fine-needle aspiration cytology and core needle biopsy under sonography or CT guidance, open surgical biopsy and video assisted thoracoscopic surgery.[2] CT guided lung biopsy was first reported by Haaga.[3] The most common post- procedural complication reported worldwide is pneumothorax followed by pulmonary haemorrhage, haemoptysis and haemothorax. Complications like systemic air embolism, needle tract seeding and death can occur in less than $1 \%$ of cases.[2,5]

In our study we studied the diagnostic yield and complications of CT guided lung lesion biopsy in lesions not amenable for either bronchoscopic or sonographic biopsy. Diagnostic yield of core needle biopsy is higher $92.4 \%$ than fine needle aspiration cytology $88 \%$.[4] Similarly, in our study the diagnostic yield with $18 \mathrm{G}$ coaxial semiautomatic core needle biopsy was $92.3 \%$. Eighteen gauge needle had similar yield and similar complication rate compared to smaller needles in CT guided lung mass biopsy.[4,6] However, 18 G needle has better maneuverability compared to smaller needles and if needed it can accommodate 0.035" guidewire catheter drainage of pneumothorax using same puncture.

Pneumothorax is the most common complication of $\mathrm{CT}$ guided lung biopsy reported in $17-26.6 \%$ of patients. Moreover, incidence of intercostal drainage in post lung biopsy pneumothorax was $0.5-38 \%$ which was comparable to our study.[5,7] Increased risk factors for post biopsy pneumothorax included chronic obstructive pulmonary disease, depth of lesion from pleura, angle of needle and fissural transgression.[3,7] In our study, Pneumothorax occurred eight patients (28\%) out of which five $(17 \%)$ were minimal pneumothorax which did not progressed on repeat scan at five minutes. One patient had mild pneumothorax which was aspirated using coaxial needle in the same sitting and check chest radiography after four hours showed no pneumothorax. However, two patients (7.1\%) developed massive pneumothorax which needed drainage and needed overnight observation. Those patients had lesions deeper than three $\mathrm{cm}$ from the pleural surface.

Pulmonary haemorrhage were seen in check CT scan in 13 patients. However, only two of them had significant hemoptysis which needed medical management. No haemothorax was observed in our study.

Pulmonary haemorrhage after $\mathrm{CT}$ guided lung biopsy was reported in $4-27 \%$ and is the second most common complication.[8,9] Clinically significant pulmonary haemorrhage with haemotysis occured in $14.3 \%$ comparable to the above mentioned studies. Risk factors for pulmonary haemorrhage are small lesion size and greater lesion depth.[10,11] Pulmonary artery hypertension also contributes to risk of pulmonary haemorrhage.[5] Most of the pulmonary haemorrhage evidenced by post biopsy perilesional ground glass opacities ( GGO) are self limiting. Significant haemorrhage manifesting as haemoptysis should be treated with intravenous antifibrinolytics like tranexamic acid and making biopsied lung dependent to prevent aspiration of blood to other side.[5,9] However, we did not come across any post biopsy haemothorax, air embolism or death in our study.

Sample size and lack of follow up in retrospective study is the limitation of our study. Due to which we could not study the 
factors determining the complications and incidence of needle tract seeding.

\section{CONCLUSION}

CT guided lung lesion biopsy with $18 \mathrm{G}$ coaxial semi-automatic core biopsy needle is a safe procedure with good diagnostic yield and relatively low incidence of clinically significant complications.

\section{CONFLICT OF INTEREST}

None

\section{SOURCES OF FUNDING}

None

\section{REFERENCES}

1. Spira A, Halmos B, Powell CA. Pulmonary, Sleep, and critical care update in Lung Cancer 2015. 2016;194:661-71.

2. Chojniak R, Nicole P, Pinto V, Ting CJ, Cohen MP, Yu LS. Computed tomography-guided transthoracic needle biopsy of pulmonary nodules . 2011;44(5):315-20.

3. Li Y, Du Y, Yang HF, Yu JH, Xu XX. CT-guided percutaneous core needle biopsy for small ( $20 \mathrm{~mm}$ ) pulmonary lesions. $2013 ; 68$.

4. Loh SEK, Uk F, Wu DDF, Venkatesh SK, Uk F, Ong CK. CT-Guided Thoracic Biopsy: Evaluating Diagnostic Yield and Complications. 2013;42(6):285-90.

5. Wu CC, Maher MM, Shepard JO, Cc W, Mm M, Jo S. Complications of CT-Guided Percutaneous Needle Biopsy of the Chest: Prevention and Management. 2011;(June):678-82.

6. Unver E, Yilmaz A, Aksoy F, Baysungur V, Genc O. Does needle size affect diagnostic yield of transthoracic needle biopsy in malignant pulmonary lesions?: Comparison of 18- , 22- and 25-gauge needles in surgical specimens. 2006;648-51.

7. Hiraki T, Mimura $\mathrm{H}$, Gobara $\mathrm{H}$, Shibamoto K, Inoue D, Matsui $\mathrm{Y}$, et al. Vascular and interventional radiology;(March):809-14.

8. Moghaddam SR, Thalhammer A. Variables affecting the risk of pneumothorax and intrapulmonal hemorrhage in CT-guided transthoracic biopsy. 2008;1356-63.

9. Mir MF, Naik MA, Malik JA. Original Research Article CT guided lung biopsy complications: a single centre prospective study. 2018;6(9):2943-7.

10. Yeow K, Su I, Pan K, Tsay P, Lui K, Cheung Y, et al. Risk Factors of Pneumothorax and Bleeding Multivariate Analysis of 660 CT-Guided Coaxial. Chest. The American College of Chest Physicians; 126(3):748-54.

11. Gohari A, Haramati LB. Complications of CT Scan- Guided Lung Biopsy Lesion Size and Depth Matter. Chest. The American College of Chest Physicians; 2003;126(3):666-8. 